\title{
Low-complexity adaptive iteration algorithm for frequency tracking in OFDM systems
}

\author{
Hong-Yu Liu* and Yung-Chang Yin
}

\begin{abstract}
The carrier frequency offset (CFO), destroying the orthogonality between subcarriers, greatly degrades the performance of an orthogonal frequency-division multiplexing system. One of the most frequently used ways for a CFO estimator design is to adopt maximum-likelihood (ML) estimation, achieving high accuracy tightly close to the Cramer-Rao lower bounds (CRLBS).

One of the ML-based algorithms, called linearly-combined CFO (LC-CFO), evaluates all the single-time-slot CFO estimates first and then linearly combined these CFO estimates in the minimum mean-square-error sense. Its tracking range is quite wide up to half the carrier spacing, and convergence speed is very fast, costing only several tens of iterations; moreover, its mean-square error (MSE) performances are very much close to CRLBs at medium-to-high signal-to-noise ratio (SNR) values. However, a set of arctangent functions is needed to be evaluated for each iteration, which increases the computational complexity. In this article, a low complexity, called simplified LC-CFO (SLC-CFO), is proposed that the set of arctangent functions are replaced by low-complexity limiters, resulting in simplifying the receiver design and reducing the computational load while keeping nearly the same tracking range and MSE performances. With proper choice of a parameter, SLC-CFO even shows faster convergence speed and lower MSE value at low SNR, compared with LC-CFO. Simulation results demonstrate all these aforementioned properties.
\end{abstract}

Keywords: Orthogonal frequency-division multiplexing, Carrier frequency offset, Maximum-likelihood estimation, Channel estimation, Linear minimum mean-square error

\section{Introduction}

Orthogonal frequency-division multiplexing (OFDM) transforms a frequency-selective multipath fading channel into multiple frequency-flat channels that makes appealing for designing a receiver using simple signal processing techniques [1]. The orthogonality between subcarriers, allowing overlapping between sub-bands without introducing inter-channel interference, provides high spectral efficiency. However, the existence of carrier frequency offset (CFO) due to the Doppler spread and/or the instability of the local oscillators destructs the orthogonality, resulting in serious performance degradation [2-4]. Therefore, the demand for CFO estimation with high accuracy is essential to OFDM systems.

\footnotetext{
* Correspondence: hongyu.liu@msa.hinet.net

Department of Electrical Engineering, Fu Jen Catholic University, No. 510,

Zhongzheng Rd., Xinzhuang Dist., New Taipei City 24205, Taiwan (Republic of China)
}

There are many correlation-based algorithms proposed for CFO estimation in [5-9]. They are proved useful for CFO acquisition, acquiring coarse CFO estimates. To secure CFO estimates with high accuracy, the technique using maximum-likelihood (ML) estimation perhaps is the best choice for CFO tracking, securing fine CFO estimates. In [10-13], the ML CFO estimators are developed. However, they can only work for systems employing repeated preambles. The others proposed in [14-20] have no such a limitation. Applying ML estimation for estimating CFO is usually cumbered by the nonlinear nature of the likelihood function. The problem of local extremum or multiple solutions arises. Many papers resort to making approximation by truncating high order terms of a Taylor series [14-16, 18-20]. Recently, some research papers consider the inclusion of phase noise when performing CFO estimation [21, 22]. The source of phase noise is the instability of local oscillators, which
Springer

(c) 2016 Liu and Yin. Open Access This article is distributed under the terms of the Creative Commons Attribution 4.0 International License (http://creativecommons.org/licenses/by/4.0/), which permits unrestricted use, distribution, and reproduction in any medium, provided you give appropriate credit to the original author(s) and the source, provide a link to the Creative Commons license, and indicate if changes were made. 
can be modeled as time-varying correlated noise [23]. In [21], the truncation of high order Taylor series is used for acquiring the approximate CFO estimate. One of the two proposed methods in [22] must resort to exhaustive search for CFO estimation, and the other is correlation based, which is applicable to the systems employing repeated preambles. The closed-form formula for the ML CFO estimate without carrying out Taylor series truncation is reported in [17], where the closed-form solutions to all the single-time-slot samples are first found and then those estimates are linearly combined in the sense of minimum mean-square error (MSE). Thus, we call it linearly combined CFO (LC-CFO) estimator. The conducted simulations for it show that the MSE performances of the CFO estimator are very much close to the Cramer-Rao lower bounds (CRLBs).

In this article, we develop a low-complexity CFO estimator, called simplified LC-CFO (SLC-CFO), adapted from LC-CFO developed in [17]. The main idea of SLCCFO having a low complexity lies in replacing a set of arctangent functions in LC-CFO by simple, low-complexity limiters. As for the implementation issue of an arctangent function, the use of look-up table stored in memory perhaps is attractive for high-speed arctangent computation. However, this simple method requires high amount of memory to provide accuracy. In [24], the rational approximation for an arctangent function is proposed. Dividers and multipliers are needed for the implementation. The circuit of coordinate rotation digital computer [25] is another choice for angle estimation. However, the quantization issue affecting the precision [26] must be considered. Since no arctangent functions are required in our proposed method, the receiver design is simplified and requires less computational loading. The effects on the replacement with limiters will be thoroughly discussed and examined by computer simulation. The tracking range, the convergent speed, and the MSE for CFO estimation as well as channel impulse response (CIR) estimation are included.

The rest of the article is organized as follows. Section 2 introduces the system model and derives the singe-timeslot CFO estimator. Section 3 reviews the LC-CFO algorithm. In Section 4, SLC-CFO, adapted from LC-CFO, is developed. Simulation results are then presented in Section 5, showing the advantages of SLC-CFO with detailed discussions. Finally, conclusions are drawn in Section 6.

\section{System model and single-time-slot CFO estimator} Consider an OFDM system with $N$ subcarriers. Let $X_{k}$ be the transmitted $k$ th subcarrier symbol, $H_{k}$ be the $k$ th subcarrier frequency channel response, $k=0,1, \cdots, N-1$, and $\delta$ be the carrier frequency offset normalized to the carrier spacing. At the receiver, after discarding the cyclic prefix, the complex baseband received signal at the $n$th time slot, $n=0,1, \cdots, N-1$, in an OFDM block can be expressed as

$$
r_{n}=e^{j 2 \pi n \delta / N} y_{n}+w_{n}
$$

where $w_{n}$ is the additive white Gaussian noise with zero mean and variance $\sigma_{w}^{2}$ at the $n$th time slot sample and

$$
y_{n}=\frac{1}{\sqrt{N}} \sum_{k=0}^{N-1} H_{k} X_{k} e^{j 2 \pi n k / N}
$$

is the noise-free, CFO-free received signal at the $n$th time slot.

The ML estimator of $\delta$ for each time slot sample in (1) can be obtained as [17]

$$
\hat{\delta}_{n}=\frac{N}{2 \pi n}\left(\tan ^{-1}\left\{\frac{\operatorname{Im}\left\{r_{n} y_{n}^{*}\right\}}{\operatorname{Re}\left\{r_{n} y_{n}^{*}\right\}}\right\}+\varepsilon_{n}\right),
$$

where $\operatorname{Re}\{\cdot\}$ and $\operatorname{Im}\{\cdot\}$ mean real part and imaginary part, respectively, and $\varepsilon_{n}$ is defined as

$$
\varepsilon_{n}=\left\{\begin{array}{cc}
0 & \text { when } \operatorname{Re}\left\{r_{n} y_{n}^{*}\right\}>0, \\
-\pi & \text { when } \operatorname{Re}\left\{r_{n} y_{n}^{*}\right\}<0 \text { and } \operatorname{Im}\left\{r_{n} y_{n}^{*}\right\}<0, \\
\pi & \text { when } \operatorname{Re}\left\{r_{n} y_{n}^{*}\right\}<0 \text { and } \operatorname{Im}\left\{r_{n} y_{n}^{*}\right\}>0 .
\end{array}\right.
$$

Notice that the parameter $\varepsilon_{n}$ is not be included in [17]. We have slightly modified the single-time-slot CFO estimator developed in [17]. The functionality of $\varepsilon_{n}$ is explained as follows. The arctangent function $\tan ^{-1}\{\cdot\}$ in (3) represents the angle of the complex product $r_{n} y_{n}^{*}$; moreover, the range of $\tan ^{-1}\{\cdot\}$ is from $-\pi / 2$ to $\pi / 2$. Aided by the additional parameter $\varepsilon_{n}$ following $\tan ^{-1}\{\cdot\}$, the angle of $r_{n} y_{n}^{*}$ represented can be extended to the range from $-\pi$ to $\pi$. Furthermore, for the $N$ received signal samples $\left\{r_{n}, n=0,1, \cdots, N-1\right\}$ of (1), $\delta$ exists only in the $N-1$ received signal samples $\left\{r_{n}, n=1, \cdots, N-1\right\}$. The number of the single-time-slot CFO estimators of (3) is thus $N-1$.

\section{Review of the LC-CFO algorithm}

Linearly combining these $N-1$ single-time-slot estimators of (3), the LC-CFO estimator can be expressed as [17]

$$
\hat{\delta}_{L C}=\sum_{n=1}^{N-1} \alpha_{n} \hat{\delta}_{n}
$$

where 


$$
\alpha_{n}=\frac{n^{2}\left|y_{n}\right|^{2}}{\sum_{p=1}^{N-1} p^{2}\left|y_{p}\right|^{2}}
$$

is obtained from minimizing the estimate error $\Delta \delta=\hat{\delta}_{L C}-\delta$ in a linear-minimum-MSE sense.

The exact values of the noise-free, $\mathrm{CFO}$-free received signals $\left\{y_{n}, n=1,2, \cdots, N-1\right\}$ in Eqs. (3), (4), and (6) are unknown to the receiver due to the lack of the knowledge of CIR. By applying ML estimation to estimate CIR, the resulting estimator of $\mathbf{y}=\left[y_{0}, y_{1}, \cdots y_{N-1}\right]^{T}$ with the superscript $T$ denoting transpose can be readily obtained as

$$
\hat{\mathbf{y}}=\mathbf{A D}_{\delta}^{H} \mathbf{r}
$$

where

$$
\begin{aligned}
& \mathbf{A}=\frac{1}{N} \mathbf{F}_{N}^{H} \mathbf{U}_{X} \mathbf{F}_{v}\left(\mathbf{F}_{v}^{H} \mathbf{U}_{X}^{H} \mathbf{U}_{X} \mathbf{F}_{v}\right)^{-1} \mathbf{F}_{v}^{H} \mathbf{U}_{X}^{H} \mathbf{F}_{N}, \\
& \mathbf{D}_{\delta}=\operatorname{diag}\left\{e^{j 2 \pi 0 \cdot \delta / N}, e^{j 2 \pi 1 \cdot \delta / N}, \cdots, e^{j 2 \pi(N-1) \delta / N}\right\}, \\
& \mathbf{r}=\left[r_{0}, r_{1}, \cdots, r_{N-1}\right]^{T}
\end{aligned}
$$

with

$$
\mathbf{U}_{X}=\operatorname{diag}\left\{X_{0}, X_{1}, \cdots, X_{N-1}\right\}
$$

and

$$
\mathbf{F}_{v}=\left[\begin{array}{cccc}
1 & 1 & \cdots & 1 \\
1 & e^{-j 2 \pi 1 \cdot 1 / N} & \cdots & e^{-j 2 \pi 1 \cdot(v-1) / N} \\
\vdots & \vdots & \cdots & \vdots \\
1 & e^{-j 2 \pi(N-1) \cdot 1 / N} & \cdots & e^{-j 2 \pi(N-1)(v-1) / N}
\end{array}\right] .
$$

The symbol $\operatorname{diag}\{\cdot\}$ represents a diagonal matrix with its diagonal entries being inside the braces.

The parameter $\delta$ in (7) is still unknown at this present. To solve this problem, (5) and (7) are updated alternatively, which is called Version $A$ in [17]. Another algorithm, called Version $B$ in [17] or LC-CFO in this article, is to add an additional step to Version $A$ after performing the CFO estimator of (5). Since the LC-CFO algorithm is iterative such that it will eventually converge after enough rounds of iterations, we will add an additional subscript $s$ to represent the $s$ th iteration. $\mathbf{r}_{s}$, for example, represents the received signal vector $\mathbf{r}$ having been performing the sth iteration. The LC-CFO (or Version B) algorithm, in our notations, are as the following.

\section{Step 1)}

$$
\hat{\mathbf{y}}_{s}=\mathbf{A r}_{s-1}
$$

Step 2)

$$
\hat{\delta}_{L C, s}=\frac{N}{2 \pi \sum_{p=1}^{N-1} p^{2}\left|\hat{y}_{p, s}\right|} \sum_{n=1}^{N-1} n\left|\hat{y}_{n, s}\right|^{2}\left(\tan ^{-1}\left\{\frac{\operatorname{Im}\left\{r_{n, s-1} \hat{y}_{n, s}^{*}\right\}}{\operatorname{Re}\left\{r_{n, s-1} \hat{y}_{n, s}^{*}\right\}}\right\}+\varepsilon_{n, s}\right),
$$

where

$$
\varepsilon_{n, s}=\left\{\begin{array}{cc}
\text { when } \operatorname{Re}\left\{r_{n, s-1} y_{n, s}^{*}\right\}>0, \\
-\pi & \text { when } \operatorname{Re}\left\{r_{n, s-1} y_{n, s}^{*}\right\}<0 \text { and } \operatorname{Im}\left\{r_{n, s-1} y_{n, s}^{*}\right\}<0, \\
\pi & \text { when } \operatorname{Re}\left\{r_{n, s-1} y_{n, s}^{*}\right\}<0 \text { and } \operatorname{Im}\left\{r_{n, s-1} y_{n, s}^{*}\right\}>0 .
\end{array}\right.
$$

Step 3)

$$
\mathbf{r}_{s}=\mathbf{D}_{\hat{\delta}_{L C, s}^{H}}^{H} \mathbf{r}_{s-1} .
$$

$\mathbf{r}_{0}$ coming up at the first iteration is set equal to the received noisy signal $\mathbf{r}$. Notice that, at Step 3, frequency offset is corrected, and an equivalent received noisy signal $\mathbf{r}_{s}$ is produced. Then, the process goes back to Step 1 for the next iteration to estimate the residual CFO left in the equivalent received noisy signal produced in the previous iteration. In Step 1, no need to make correction of CFO as made in (7) since in the previous step, i.e., in Step 3, the CFO correction has been done. After several iterations, $\hat{\delta}_{L C, s}$ in Step 2 will approximately be zero. After a total of $L$ iterations are performed, the CFO estimate is summation of $L$ LC-CFO estimates, i.e., $\sum_{p=1}^{L} \hat{\delta}_{L C, p}$. Then, the accumulated estimate error after $s$ iterations is $\Delta \delta_{s}=\sum_{p=1}^{s} \hat{\delta}_{L C, p}-\delta, s=1,2, \cdots, L$. In addition, the ML CIR estimator can be readily to be shown as

$$
\hat{\mathbf{h}}_{s}=\frac{1}{\sqrt{N}}\left(\mathbf{F}_{v}^{H} \mathbf{U}_{X}^{H} \mathbf{U}_{X} \mathbf{F}_{v}\right)^{-1} \mathbf{F}_{v}^{H} \mathbf{U}_{X}^{H} \mathbf{F}_{N} \mathbf{r}_{s} .
$$

\section{The proposed SLC-CFO algorithm}

From the LC-CFO algorithm reviewed in the previous section, the CFO estimator $\hat{\delta}_{L C, S}$ of (14) estimates the residual CFO existing in $\mathbf{r}_{s-1}$. Here, we propose the replacement of the $N-1$ arctangent functions in (14) by $N-1$ simple limiters since the residual CFO estimator $\hat{\delta}_{L C, s}$ of (14) will approach to zero when LC-CFO converges and the fact that $\tan ^{-1} \phi_{n, s} \approx \phi_{n, s}$ when $\phi_{n, s}$ is small enough. The following describes the proposed SLC-CFO algorithm. 
Step 1)

$$
\hat{\mathbf{y}}_{s}=\mathbf{A} \mathbf{r}_{s-1} .
$$

Step 2)

$$
\hat{\delta}_{L C, s}=\frac{N}{2 \pi \sum_{p=1}^{N-1} p^{2}\left|\hat{y}_{p, s}\right| 2} \sum_{n=1}^{N-1} n\left|\hat{y}_{n, s}\right|^{2} \phi_{n, s},
$$

Where

a) $\phi_{n, s}=\operatorname{Im}\left\{r_{n, s-1} \hat{y}_{n, s}^{*}\right\} / \operatorname{Re}\left\{r_{n, s-1} \hat{y}_{n, s}^{*}\right\}$ when $\operatorname{Re}\left\{r_{n, s-1} \hat{y}_{n, s}^{*}\right\}$ $>0$ and $-\lambda \leq \operatorname{Im}\left\{r_{n, s-1} \hat{y}_{n, s}^{*}\right\} / \operatorname{Re}\left\{r_{n, s-1} \hat{y}_{n, s}^{*}\right\} \leq \lambda$;

b) $\phi_{n, s}=-\lambda$ when $\operatorname{Re}\left\{r_{n, s-1} \hat{y}_{n, s}^{*}\right\}>0$ and Im $\left\{r_{n, s-1} \hat{y}_{n, s}^{*}\right\} / \operatorname{Re}\left\{r_{n, s-1} \hat{y}_{n, s}^{*}\right\}<-\lambda$, or when $\operatorname{Re}$ $\left\{r_{n, s-1} \hat{y}_{n, s}^{*}\right\}<0$ and $\operatorname{Im}\left\{r_{n, s-1} \hat{y}_{n, s}^{*}\right\}<0$;

c) $\phi_{n, s}=\lambda$ when $\operatorname{Re}\left\{r_{n, s-1} \hat{y}_{n, s}^{*}\right\}>0$ and $\operatorname{Im}\left\{r_{n, s-1} \hat{y}_{n, s}^{*}\right\} /$ $\operatorname{Re}\left\{r_{n, s-1} \hat{y}_{n, s}^{*}\right\}>\lambda$, or when $\operatorname{Re}\left\{r_{n, s-1} \hat{y}_{n, s}^{*}\right\}<0$ and $\operatorname{Im}\left\{r_{n, s-1} \hat{y}_{n, s}^{*}\right\}>0$.

Step 3)

$$
\mathbf{r}_{s}=\mathbf{D}_{\hat{\delta}_{L C, s}^{H}}^{H} \mathbf{r}_{s-1} .
$$

The modifications to the LC-CFO algorithm lie in Step 2, resulting in the SLC-CFO algorithm with much low computational requirements. An additional parameter $\lambda$, acting as a threshold value for the $N$ limiter functions $\left\{\phi_{n, s}, n=1\right.$, $2, \cdots, N-1\}$, is introduced since the discrepancy between $\tan ^{-1} \phi_{n, s}$ and $\phi_{n, s}$ gets larger when the absolute value of $\phi_{n, s}$ grows larger. This is illustrated in Fig. 1. Thus, we introduce the parameter $\lambda$, limiting the maximum value change at every iteration, to prevent the algorithm from divergence. In addition, during the first several iterations or at a low signal-to-noise ratio (SNR) environment, the noise-free, CFO-free received estimator $\hat{\mathbf{y}}_{s}$ is still unreliable, making what follows, the CFO estimator $\hat{\delta}_{L C, s}$, departs form the residual CFO existing in $\mathbf{r}_{s-1}$. This is why we need a limiter with two threshold values of the upper limit $\lambda$ and the lower limit $-\lambda$. As for the choice of $\lambda$, it will be thoroughly discussed in the next section.

Regarding the implementation issues of the arctangent function in (14), many researchers have suggested the CORDIC-related algorithms [1, 27], which is far more complex than the limiter that we replace with.

The CIR estimator remains the same as described in (17) without modifying. When the Zadoff-Chu sequences $[28,29]$ with constant amplitudes are used for training symbols, (17) can be further simplified to

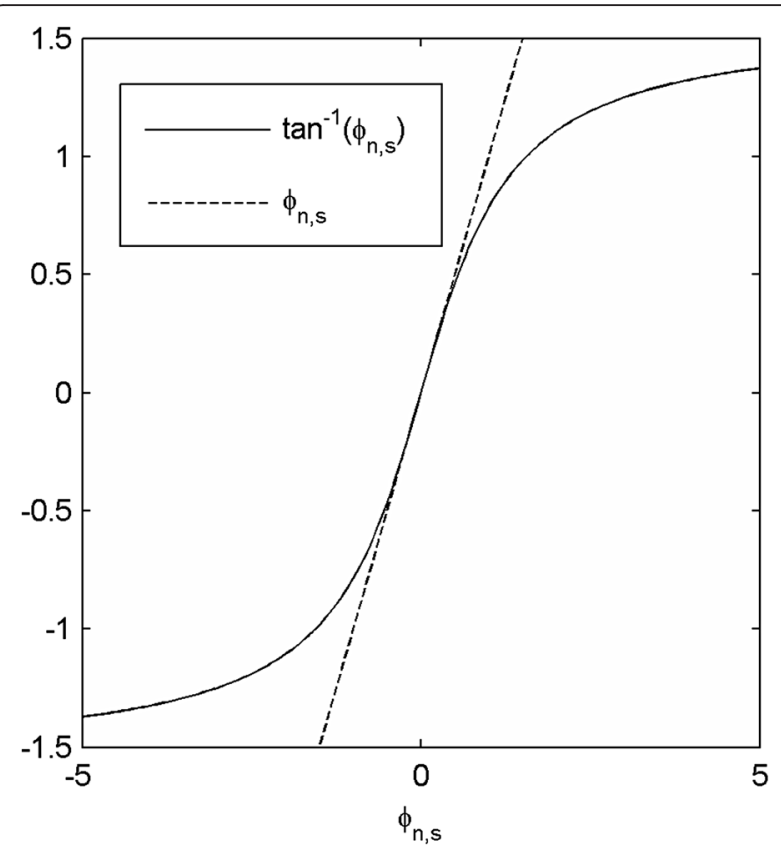

Fig. 1 Arctangent function and its Taylor series function of order one

$$
\hat{\mathbf{h}}_{s}=\frac{1}{N \sqrt{N}} \mathbf{F}_{\nu}^{H} \mathbf{U}_{X}^{H} \mathbf{F}_{N} \mathbf{r}_{s} .
$$

The facts that $\mathbf{U}_{X}^{H} \mathbf{U}_{X}=\mathbf{I}_{N}$ and $\mathbf{F}_{v}^{H} \mathbf{F}_{v}=N \mathbf{I}_{N}$, with $\mathbf{I}_{N}$ denoting an identity matrix, have been used for the CIR estimator of (21).

Notice that the proposed SLC-CFO as well as the original LC-CFO is in fact the joint estimation of CFO and CIR. The CIR information is hidden in the CFO-free, noise-free received signal vector $\mathbf{y}$ such that $\mathbf{y}=\frac{1}{\sqrt{N}} \mathbf{F}_{N}^{H}$ $\mathbf{U}_{X} \mathbf{F}_{v} \mathbf{h}$. In each of the iterative round of (18) as well as (13), the temporary CIR estimate is implicitly evaluated. The reason why we do not explicitly show CIR in both the SLC-CFO and the LC-CFO is that we focus on the simplification of the iterative CFO estimator instead of the CIR estimator. The transient behavior of CIR estimates is of no importance. Only the final (or steady-state) CIR estimate is inspected using (21) in our simulations.

\section{Simulation results and discussions}

We evaluate the OFDM systems with the number of subcarriers $N=64$. The CIR of length 9 is defined as $h_{n}$, $n=0,1, \cdots, 8$. Both a static and a Rayleigh fading channels are considered. The two simulated channel models have the same power delay profile of $E\left\{\left|h_{n}\right|^{2}\right\}=a e^{-n / 4}$, where $E\{\cdot\}$ denotes mathematical expectation, $a$ is for power normalization to unity and $n=0,1, \cdots, 8$. All the simulated results are averaged over 2000 runs for the static channel and 20000 runs for the Rayleigh fading 
one. One of the Zadoff-Chu sequences $\left\{X_{k}=e^{j \pi 7 k^{2} / N}\right.$, $k=0,1, \cdots, N-1\}$ is chosen as the training symbols for both LC-CFO and SLC-CFO.

The static channel is first used to demonstrate the merits of SLC-CFO over LC-CFO and explore the properties of convergence speed and the estimator MSE. Figure 2 shows the learning curves of $\mathrm{CFO}$ estimators for $\mathrm{LC}-\mathrm{CFO}$ and SLC-CFO at various $\mathrm{CFO}$ values. The threshold value of $\lambda$ is set 0.5 for SCL-CFO. The results show us that the larger the CFO values, the slower the convergence speed as expected since more iterations are required for approaching the estimated $\mathrm{CFO}$ values. Also notice that SLC-CFO represents slightly faster convergence speed especially at larger CFO than LC-CFO. More details are unveiled in Figs. 3 and 4 for SLC-CFO at various threshold values and different CFOs. For $\delta=$ 0.2 , SLC-CFO and LC-CFO have the same convergence speed when $\lambda$ is greater than or equal to 1 , shown in Fig. 3. For a larger CFO of $\delta=0.5$, SLC-CFO shows faster convergence than LC-CFO when $\lambda$ is slightly greater than 1 as shown in Fig. 4. These results demonstrate that the larger the threshold value of $\lambda$, the faster the convergence speed, which is obvious for large CFO values.

For Figs. 3 and 4, the SNR is set at $20 \mathrm{~dB}$. The results of CFO estimator MSE as well as CIR estimator MSE for various SNR values are shown in Figs. 5 and 6 for $\delta=0.2$ and Figs. 7 and 8 for $\delta=0.5$, where the Cramer-Rao lower bound (CRLB) is added for each of the figures for comparisons. The derivations for CRLB can be referred

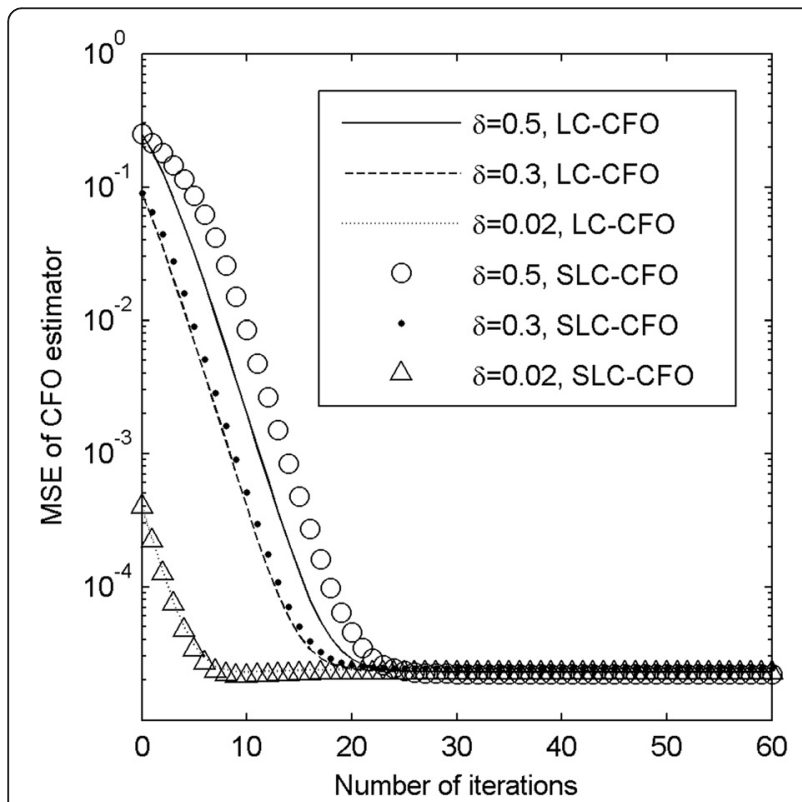

Fig. 2 Learning curves of the CFO estimator MSE for LC-CFO and SLC-CFO at various $\delta$ with $\mathrm{SNR}=20 \mathrm{~dB} . \lambda=0.5$ for SLC-CFO

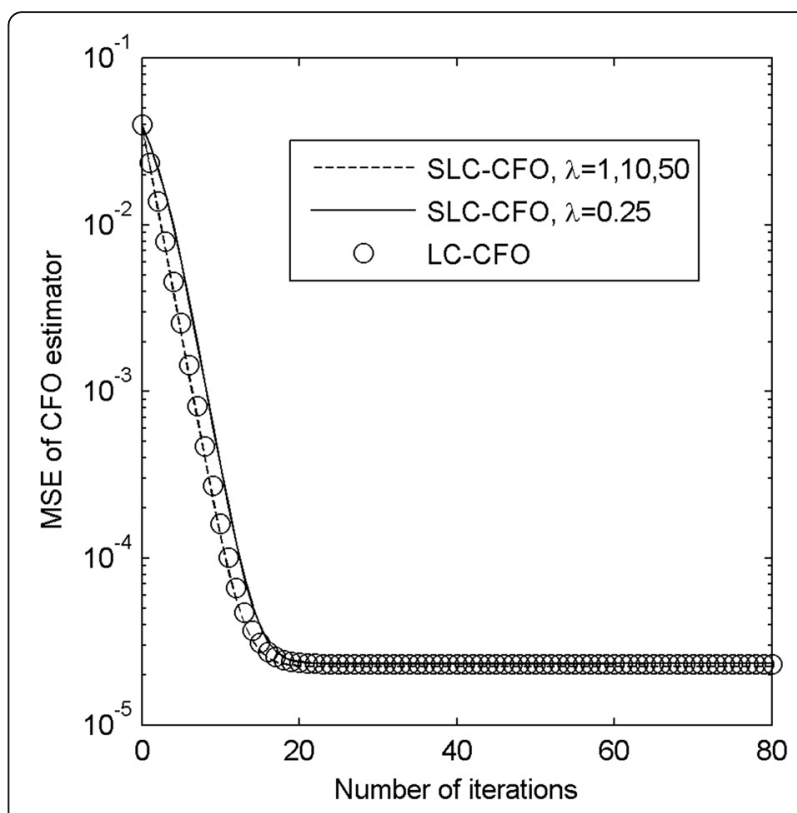

Fig. 3 Learning curves of the CFO estimator MSE for SLC-CFO, compared with LC-CFO, at various $\lambda$ with $S N R=20 \mathrm{~dB}$ and $\delta=0.2$

to [17]. We can observe that the curves of SLC-CFO and LC-CFO are tightly close at medium-to-high SNR values. At low SNR, choosing large $\lambda$, e.g., $\lambda=10$ or 50 , results in worse MSE performance than LC-CFO. A smaller threshold value of $\lambda$, e.g., $\lambda=1$, presents better MSE performance than LC-CFO. The property is much more obvious in CFO estimator MSE than in CIR

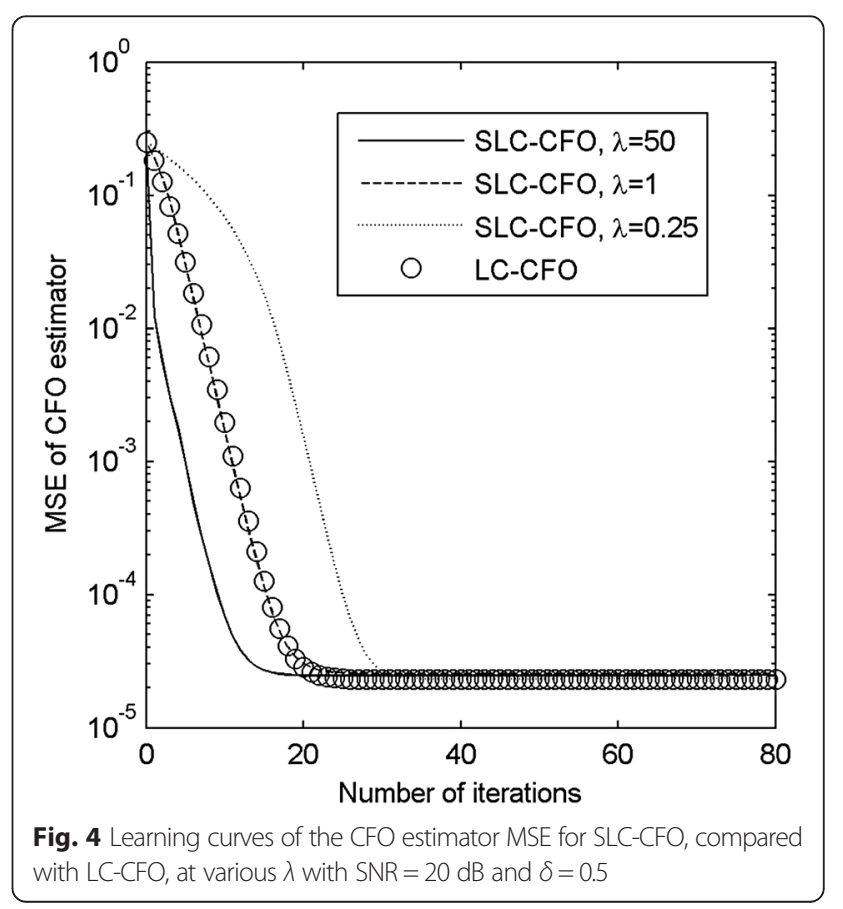




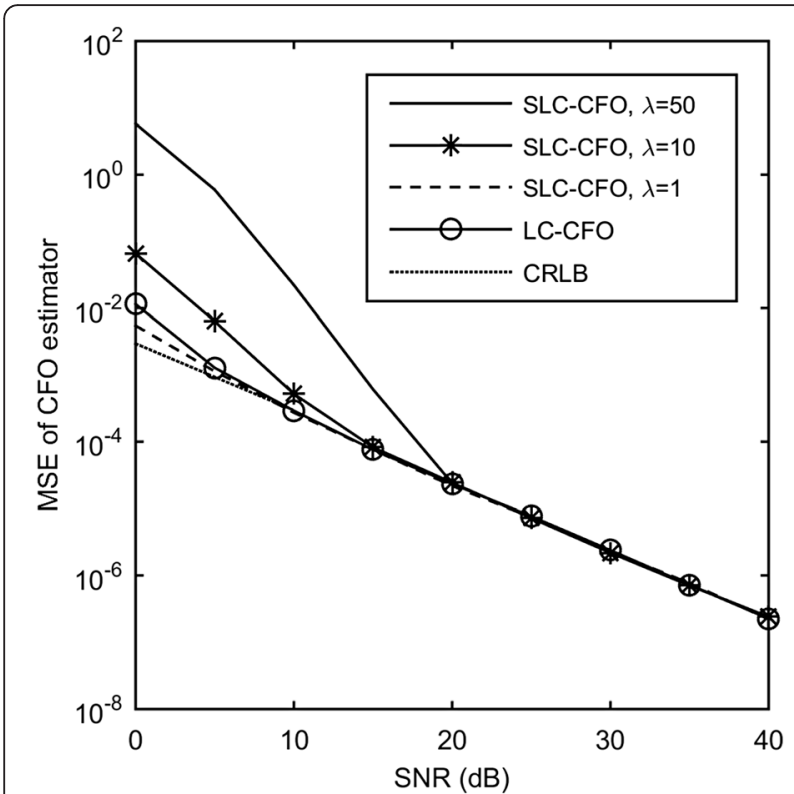

Fig. 5 CFO estimator MSE versus SNR for SLC-CFO, compared with $\mathrm{LC}-\mathrm{CFO}$, at various $\lambda$ and $\delta=0.2$

estimator MSE. The reason that the large $\lambda$ brings about the large MSE value especially at low SNR is quite apparent that a fast tracking estimator will follow closely to the random noise in a noise-dominant environment, greatly degrading estimation accuracy.

Simulations for the channel with frequency-selective Rayleigh fading are shown in Figs. 9 and 10. We observe that $\lambda=1$ has the best performance among others, and

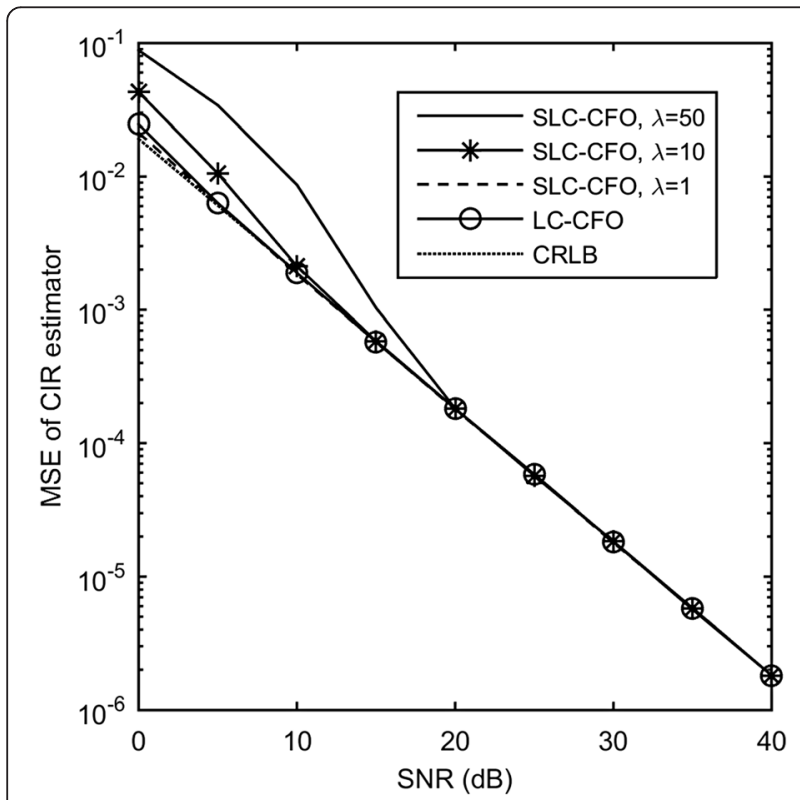

Fig. 6 CIR estimator MSE versus SNR for SLC-CFO, compared with LC-CFO, at various $\lambda$ and $\delta=0.2$

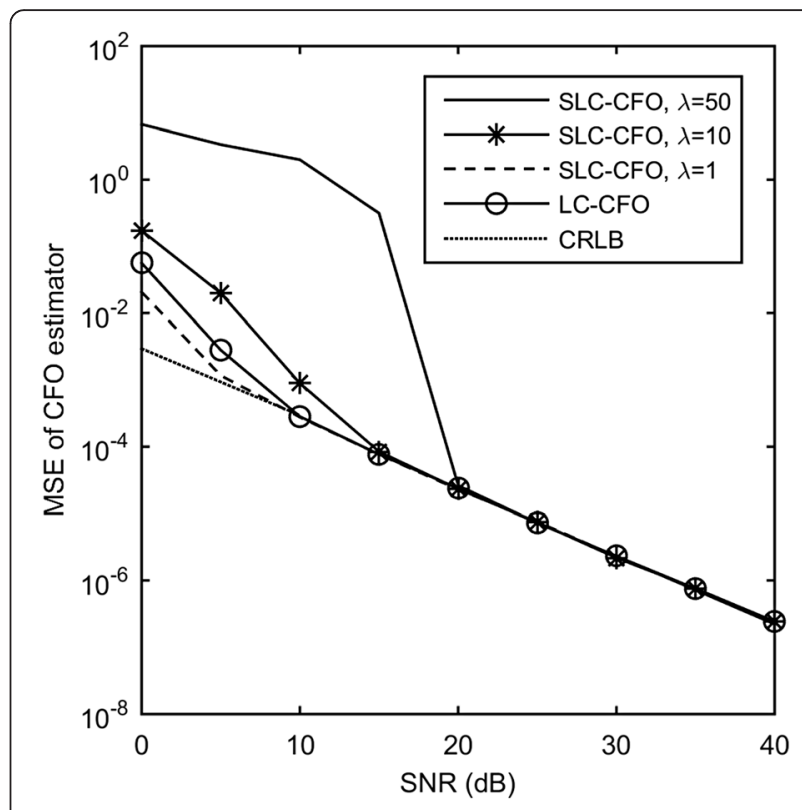

Fig. 7 CFO estimator MSE versus SNR for SLC-CFO, compared with LC-CFO, at various $\lambda$ and $\delta=0.5$

$\lambda=50$ presents the worst case. With proper choice of $\lambda$, all simulations show that the low-complexity SLC-CFO presents fast convergence speed and low MSE performance.

After several more simulations are conducted, we conclude that the best choice of $\lambda$ with both faster convergence speed and lower MSE than LC-CFO is somewhere between 1 and 3 . The number of iterations required for

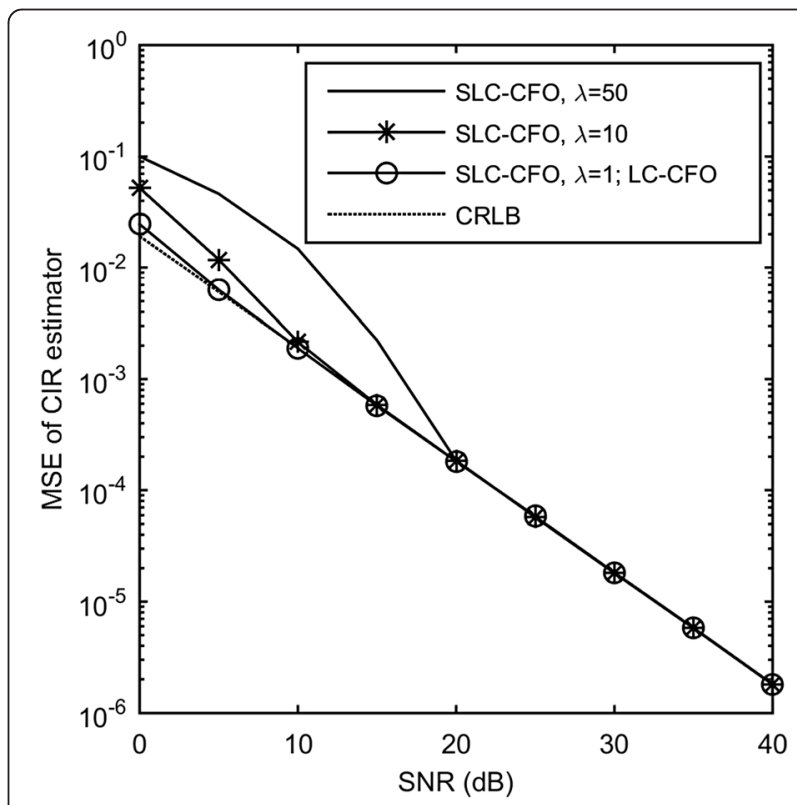

Fig. 8 CIR estimator MSE versus SNR for SLC-CFO, compared with LC-CFO, at various $\lambda$ and $\delta=0.5$ 


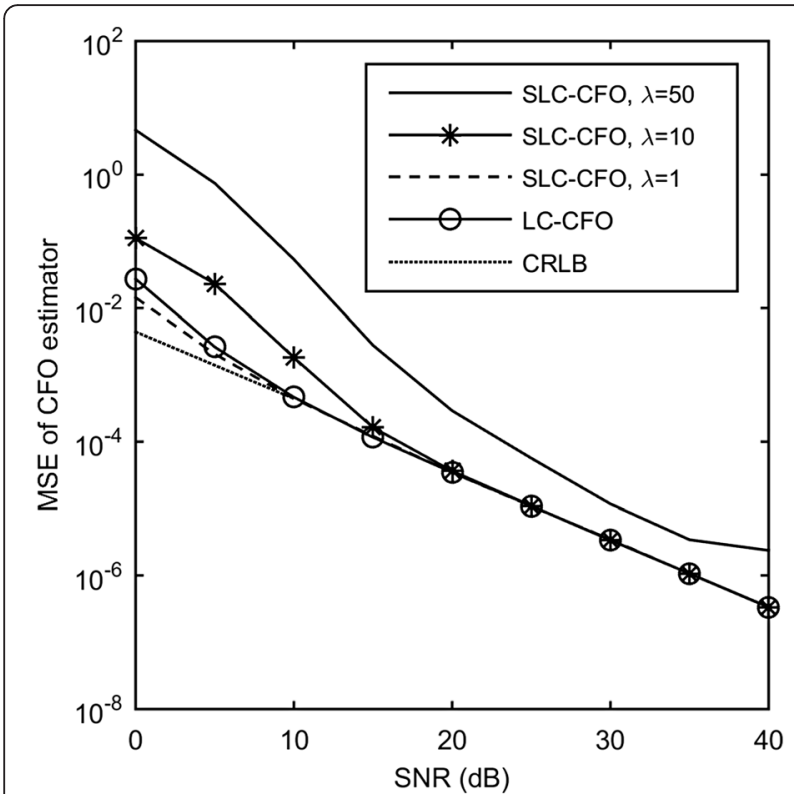

Fig. 9 CFO estimator MSE versus SNR for SLC-CFO, compared with LC-CFO, at various $\lambda$ and $\delta=0.2$ under Rayleigh fading

convergence is around 20 for $\mathrm{CFO}$ up to 0.5 . When the value of $\lambda$ chosen is several tens, the convergence rate may be faster. However, its MSE will depart from the CRLB when the signal-to-noise ratio (SNR) is lower than $20 \mathrm{~dB}$.

Finally, we will compare SLC-CFO with the existing method proposed by Salim et al. [21] because the Salim's method is one of the most competing methods. In [21], a joint estimation of channel, phase noise, and frequency

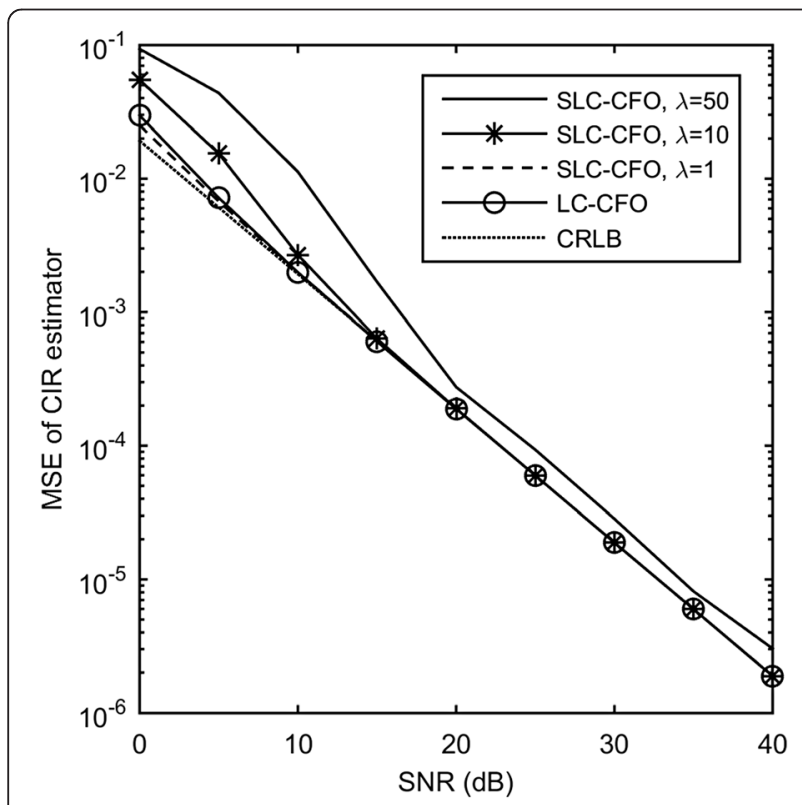

Fig. 10 CIR estimator MSE versus SNR for SLC-CFO, compared with $\mathrm{LC}-\mathrm{CFO}$, at various $\lambda$ and $\delta=0.2$ under Rayleigh fading

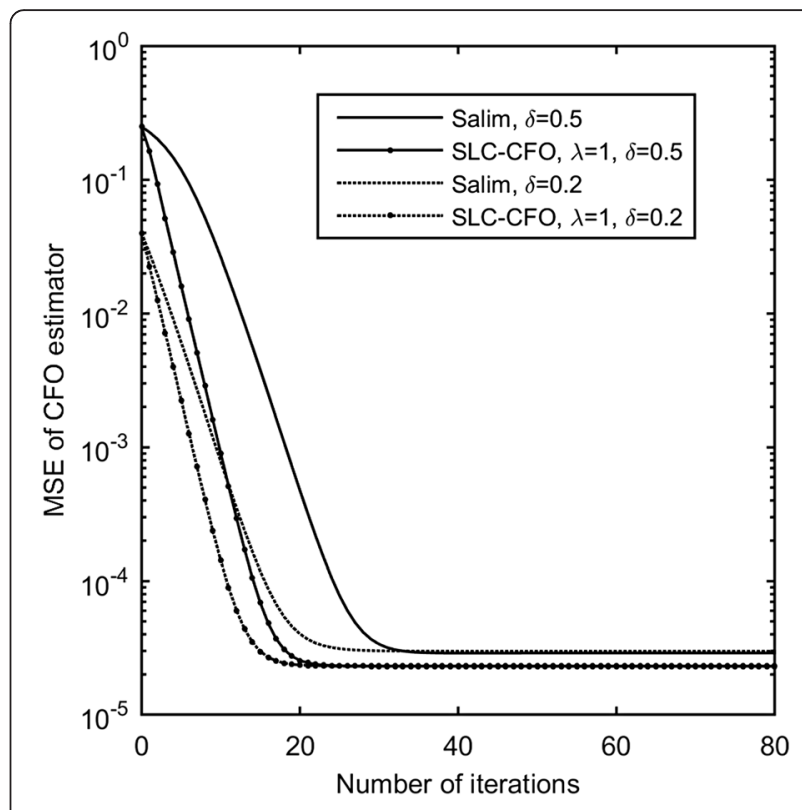

Fig. 11 Learning curves of the CFO estimator MSE for SLC-CFO, compared with Salim, for $\delta=0.2$ and 0.5 at $S N R=20 \mathrm{~dB}$

offset is considered. To make fair comparisons, no phase noise resulting from the instability of local oscillators is assumed, and the initial value of the CFO estimate is set zero. In Fig. 11, the learning curves of SLC-CFO and Salim are plotted for $\delta=0.2$ and 0.5 at $\mathrm{SNR}=20 \mathrm{~dB}$. The same static channel as adopted in the previous simulations is used. The results show that SLC-CFO has faster convergence rate and lower steady state MSEs,

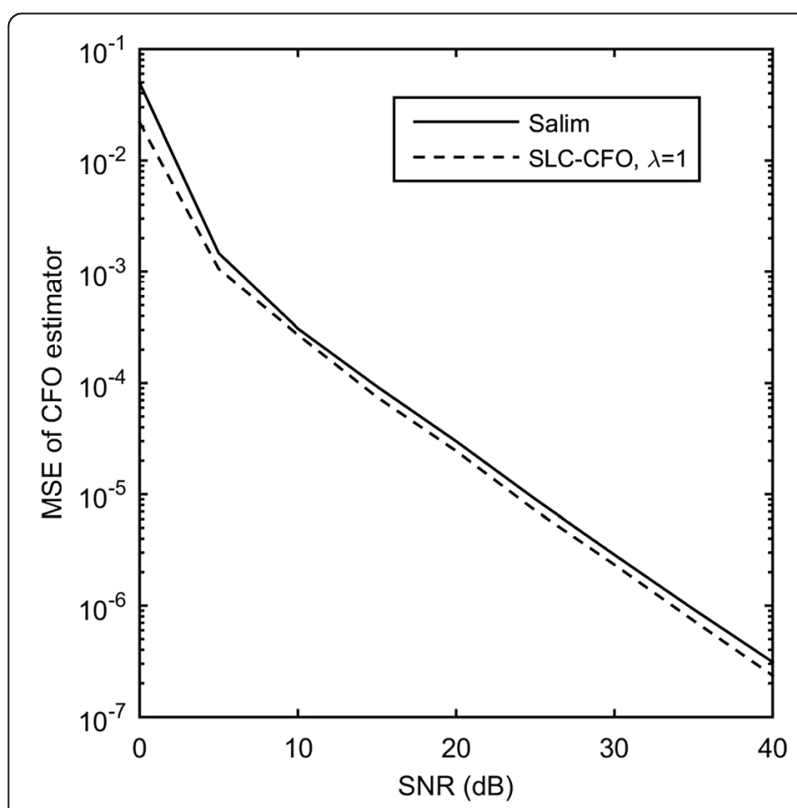

Fig. 12 CFO estimator MSE versus SNR for SLC-CFO, compared with Salim, at $\delta=0.5$ 


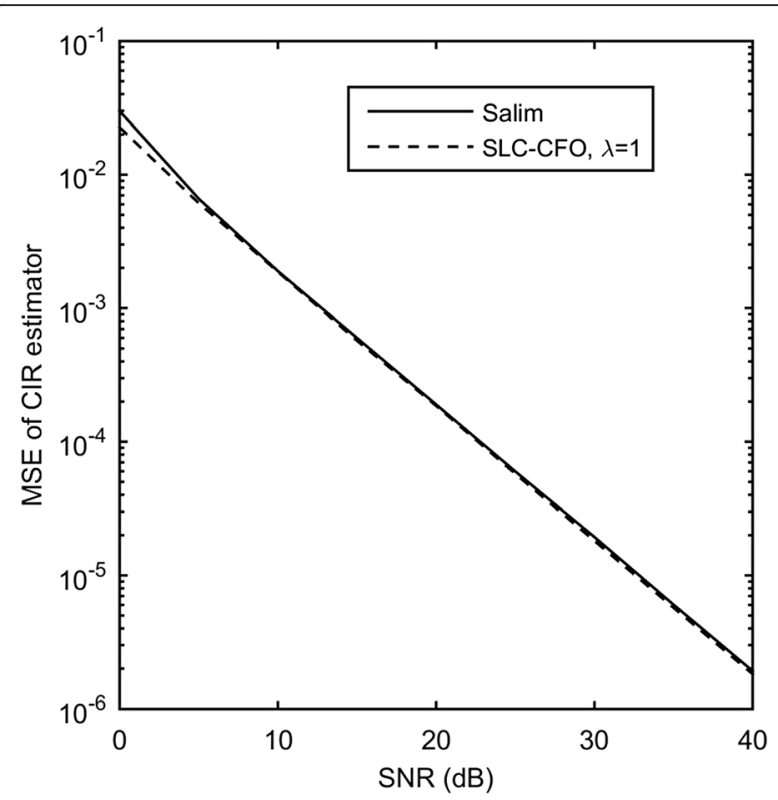

Fig. 13 CIR estimator MSE versus SNR for SLC-CFO, compared with Salim, at $\delta=0.5$

compared to the Salim's method. For $\delta=0.5$, the convergence rate of SLC-CFO is 10 iterations faster than that of Salim's method, while, for $\delta=0.2$, SLCCFO is 5 iterations faster than the Salim's method. In Figs. 12 and 13, the steady state CFO estimator MSEs and CIR estimator MSEs for SNR values from 0 to $40 \mathrm{~dB}$ are shown, respectively. Apparently, SLC-CFO has lower steady state CFO estimator MSEs than the Salim's method, while the CIR estimator MSEs are almost the same for both the methods except at low SNR values.

\section{Conclusions}

High accuracy for CFO estimation is essential for OFDM systems. Low complexity is one of the critical issues considered for implementation. We have proposed a low-complexity SLC-CFO algorithm adapted from LC-CFO with high accuracy. $N-1$ simple limiters are adopted for each iteration instead of $N-1$ arctangent functions used in LC-CFO. The computational complexity is thus reduced. By proper choice of a parameter for the limiters, not only the MSE values of the both CFO and CIR estimators at medium-tohigh SNR values maintain the same as those of LCCFO, but the convergence speed and the MSE values at low SNR values can also be improved. A static channel and a Rayleigh fading one are employed for demonstrating those properties. In brief, without sacrificing the estimator performances, a new efficient algorithm is proposed.

\section{Abbreviations}

CFO: carrier frequency offset; CIR: channel impulse response; CRLB: Cramer-Rao lower bounds; LC-CFO: linearly-combined carrier frequency offset; ML: maximum likelihood; MSE: mean-square error; OFDM: Orthogonal frequency-division multiplexing; SLC-CFO: simplified linearly-combined carrier frequency offset; SNR: signal-to-noise ratio.

Competing interests

The authors declare that they have no competing interests.

\section{Acknowledgements}

The authors would like to thank the Ministry of Science and Technology,

Taiwan, for the support of the research grant (MOST 104-2221-E-030-005-).

Received: 16 August 2015 Accepted: 10 February 2016

Published online: 20 February 2016

\section{References}

1. TD Chiueh, PY Tsai, IW Lai, Baseband Receiver Design for Wireless MIMOOFDM Communications, 2nd edn. (Wiley, Singapore, 2012)

2. T Pollet, MV Bladel, M Moeneclaey, BER sensitivity of OFDM to carrier frequency offset and Wiener phase noise. IEEE Trans. Commun. 43(2), 191-193 (1995)

3. T Keller, L Piazzo, P Mandarini, L Hanzo, Orthogonal frequency division multiplex synchronization techniques for frequency-selective fading channels. IEEE J. Sel. Areas Commun. 19(6), 999-1008 (2001)

4. M Morelli, CCJ Kuo, MO Pun, Synchronization techniques for orthogonal frequency division multiple access (OFDMA): a tutorial review. Proc. IEEE 95(7), 1394-1427 (2007)

5. $\mathrm{PH}$ Moose, A technique for orthogonal frequency division multiplexing frequency offset correction. IEEE Trans. Commun. 42(10), 2908-2914 (1994)

6. JJ van de Beek, M Sandeell, PO Borjesson, ML estimation of time and frequency offset in OFDM systems. IEEE Trans. Signal Process. 45(7), 1800-1805 (1997)

7. TM Schmidl, DC Cox, Robust frequency and timing synchronization. IEEE Trans. Commun. 45(12), 1613-1621 (1997)

8. M Morelli, U Mengali, An improved frequency offset estimator for OFDM applications. IEEE Commun. Lett. 3(3), 75-77 (1999)

9. H Minn, VK Bhargava, KB Letaief, A combined timing and frequency synchronization and channel estimation for OFDM. IEEE Trans. Commun. 54(3), 416-422 (2006)

10. $\mathrm{H}$ Yu, YT Su, Pilot-assisted maximum-likelihood frequency offset estimation for OFDM systems. IEEE Trans. Commun. 52(11), 1997-2008 (2004)

11. MH Cheng, CC Chou, Maximum-likelihood estimation of frequency and time offsets in OFDM systems with multiple sets of identical data. IEEE Trans. Signal Process. 54(7), 2848-2852 (2006)

12. H Hsieh, W Wu, Maximum-likelihood timing and carrier frequency offset estimation for OFDM systems with periodic preambles. IEEE Trans. Veh. Technol. 58(8), 4224-4237 (2009)

13. J Choi, J Lee, Q Zhao, H Lou, Joint ML estimation of frame timing and carrier frequency offset for OFDM systems employing time-domain repeated preamble. IEEE Trans. Wireless Commun 9(1), 311-317 (2010)

14. RY Yen, HY Liu, CS Tsai, Iterative joint frequency offset and channel estimation for OFDM systems using first and second order approximation algorithms. EURASIP Journal on Wireless Communication and Networking. 2012(341), 1-10 (2012)

15. RY Yen, HY Liu, CS Tsai, Finite high order approximation algorithm for joint frequency tracking and channelestimation in OFDM systems. IEICE Trans. Fundam. Electron. Commun. Comput. Sci. E95-A(10), 1676-1682 (2012)

16. RY Yen, HY Liu, YC Yin, High order approximation algorithm using GramSchmidt $Q R$ transformations coupled with an iterative correction process for tracking frequency drift in OFDM systems. EURASIP J. Wirel. Commun. Netw. 2014(16), 1-13 (2014)

17. HY Liu, RY Yen, Effective adaptive iteration algorithm for frequency tracking and channel estimation in OFDM systems. IEEE Trans. Veh. Technol. 59(4), 2093-2097 (2010)

18. JH Lee, JC Han, SC Kim, Joint carrier frequency synchronization and channel estimation for OFDM systems via the EM algorithm. IEEE Trans. Veh. Technol. 55(1), 167-172 (2006) 
19. F Merli, GM Vitetta, Iterative ML-based estimation of carrier frequency offset, channel impulse response and data in OFDM transmissions. IEEE Trans. Commun. 56(3), 497-506 (2008)

20. Y Liu, Z Tan, H Wang, KS Kwak, Joint estimation of channel impulse response and channel frequency offset for OFDM systems. IEEE Trans. Veh. Technol. 60(9), 4645-4650 (2011)

21. OH Salim, AA Nasir, H Mehrpouyan, W Xiang, S Durrani, RA Kennedy, Channel, phase noise, and frequency offset in OFDM systems: soint estimation, data detection, and hybrid Cramér-Rao lower bound. IEEE Trans. on Commun. 62(9), 3311-3325 (2014)

22. DD Lin, R Pacheco, TJ Lim, D Hatzinakos, Joint estimation of channel response, frequency offset, and phase noise in OFDM. IEEE Trans. Signal Process. 54(9), 3542-3554 (2006)

23. DD Lin, TJ Lim, The variational inference approach to joint data detection and phase noise estimation in OFDM. IEEE Trans. Signal Process. 55(5), 1862-1874 (2007)

24. R Lyons, Another contender in the arctangent race. IEEE Signal Processing Mag. 21(1), 109-110 (2004)

25. YH Hu, CORDIC-based VLSI architectures for digital signal processing. IEEE Signal Process. Mag. 9(3), 16-35 (1992)

26. $\mathrm{YH} \mathrm{Hu}$, The quantization effects of the CORDIC algorithm. IEEE Trans. Signal Process. 40(4), 834-844 (1992)

27. M Rice, Digital Communications - a Discrete-Time Approach (Pearson Prentice Hall, Upper Saddle River, 2009)

28. RL Frank, SA Zadoff, Phase shift pulse codes with good periodic correlation properties. IEEE Trans. Inf. Theory 8(6), 381-382 (1962)

29. DC Chu, Polyphase codes with good periodic correlation properties. IEEE Trans. Inform. Theory. 18(4), 531-532 (1972)

\section{Submit your manuscript to a SpringerOpen ${ }^{\mathcal{O}}$ journal and benefit from:}

- Convenient online submission

- Rigorous peer review

- Immediate publication on acceptance

- Open access: articles freely available online

- High visibility within the field

- Retaining the copyright to your article

Submit your next manuscript at $\gg$ springeropen.com 